\title{
Charge Screening in Classical Spinor Electrodynamics
}

This article has been downloaded from IOPscience. Please scroll down to see the full text article. 1991 Europhys. Lett. 16617

(http://iopscience.iop.org/0295-5075/16/7/002)

View the table of contents for this issue, or go to the journal homepage for more

Download details:

IP Address: 139.165.107.21

The article was downloaded on 05/12/2011 at 16:00

Please note that terms and conditions apply. 
Europhys. Lett., 16 (7), pp. 617-621 (1991)

\title{
Charge Screening in Classical Spinor Electrodynamics.
}

\author{
M. BAwIN and J. CUGNoN \\ Institut de Physique B5, Université de Liège - Sart Tilman, 4000 Liège 1, Belgium \\ (received 3 June 1991; accepted in final form 29 August 1991) \\ PACS. 03.50K - Other special classical field theories. \\ PACS. 11.10L - Nonlinear or nonlocal theories and models.
}

\begin{abstract}
We study the problem of a fixed source of charge $Z$ in electromagnetic interaction with a classical Dirac field. We find that partially screened solutions with energy less than the energy associated with pure Coulomb configurations (with zero spinor field) exist for $Z>Z_{\mathrm{c}}$, where $Z_{\mathrm{c}}$ is the usual value at which the eigenvalue from the (linear) Dirac equation reaches the lower continuum. In contrast with the corresponding problem of coupling to a scalar (KleinGordon) field, such solutions do not arise for $Z_{0}<Z<Z_{\mathrm{c}}$, where $Z_{0}$ is the value at which the Dirac equation has a zero eigenvalue, so that nonlinear effects of the kind considered here do not impair the usual interpretation of the Dirac equation as a single-particle equation for $Z<Z_{\mathrm{c}}$.
\end{abstract}

We recently studied $[1,2]$ the problem of strongly charged sources in classical scalar electrodynamics, i.e. we investigated the stability properties of a prescribed external source of charge $Z$ in electromagnetic interaction with a charged scalar field. We found the following results $[1,2]$ :

1) There exists a critical value $Z=Z_{\mathrm{c}}$ above which partial screening of the external source takes place. These configurations have a lower energy than the pure Coulomb configuration (with zero scalar field). The value $Z=Z_{\mathrm{c}}$ is exactly the value at which the Klein-Gordon equation for a scalar particle of mass $m$ in the external Coulomb field of the source has a bound-state energy $\omega=-m$.

2) Partially screened solutions with total energy less than the pure Coulomb solutions also exist for $Z_{0} \leqslant Z \leqslant Z_{\mathrm{c}}$, where $Z_{0}$ is the value at which the corresponding Klein-Gordon equation has a solution with $\omega=0$. Although such solutions are not expected to be stable [2], they nevertheless imply the breakdown of the interpretation of the Klein-Gordon equation as a single-particle wave equation already for $Z>Z_{0}$.

The purpose of this work is to study to what extent the above results remain true in classical spinor electrodynamics, i.e. for the problem of an external source coupled to a classical charged Dirac field. Although the usefulness of the notion of a classical Dirac field is certainly not obvious, such classical Dirac fields have been and still are being studied in connection not only with atomic physics [3], but with other fields of physics as well [4]. (References [3] and [4] contain an extensive bibliography on this subject.) Hopefully, studying the properties of a classical field theory may shed some light on the corresponding 
quantum field theory, even for Dirac fields. Aside from these general considerations, our own motivation to investigate strongly charged sources in electromagnetic interaction with classical Dirac fields rests with the ongoing difficulty in elucidating the origin of electronpositron $\left(\mathrm{e}^{-} \mathrm{e}^{+}\right)$peaks in the Darmstadt heavy-ion collision experiments [5]. In particular, we wanted to compute the $Z$-value at which the nonlinear theory implies a breakdown of the Dirac equation as a single-particle equation. The occurrence of such a breakdown for $Z_{0} \leqslant Z<Z_{\text {c }}$ (as in the Klein-Gordon case) would indeed be of possible importance in connection with the Darmstadt experiments, as $\left(\mathrm{e}^{-} \mathrm{e}^{+}\right)$peaks are known to be present for $Z<Z_{\mathrm{c}}$ [5]. A further motivation of this work is that, unlike the Klein-Gordon equation, the Dirac equation does not develop complex eigenvalues for $Z>Z_{c}$, but rather complex poles on the unphysical energy sheet [6]. We are not aware of any result in the literature relating such poles to instabilities of the nonlinear theory. It is clearly of interest to establish whether this is indeed the case. Our main result is the following.

For any value of $Z$, there exist partially screened solutions to the system consisting of a fixed source of charge $Z$ in electromagnetic interaction with a classical Dirac field of mass $m$. However, such solutions appear to have a lower energy than the pure Coulomb solution (corresponding to zero spinor field) only for $Z>Z_{\mathrm{c}}$, where $Z_{\mathrm{c}}$ is the usual critical value of the (linear) Dirac equation, i.e. the value of $Z$ at which the bound-state energy reaches the lower continuum. In contrast to the Klein-Gordon case, no such solution occurs for $Z_{0}<Z<Z_{\text {c }}$. Thus, our numerical analysis is indeed consistent with the interpretation of the onset of poles on the unphysical sheet (for $Z>Z_{\mathrm{c}}$ ) as the signal of the instability of the classical source and the breakdown of the single-particle interpretation of the Dirac equation for $Z>Z_{\text {c }}$.

In order to see this in detail, we start with the Lagrangian $\mathscr{L}$ describing a classical Dirac field $\psi$ in electromagnetic interaction with a prescribed external source $j_{\mu}^{\text {ext }}$ :

$$
\mathscr{L}=\bar{\psi}\left(i \gamma^{\mu} \partial_{\mu}-e \gamma^{\mu} A_{\mu}-m\right) \psi-\frac{1}{4}\left(\partial_{\mu} A^{\nu}-\partial^{\nu} A_{\mu}\right)\left(\partial^{\mu} A_{v}-\partial_{\nu} A^{\mu}\right)-e j_{\mu}^{\mathrm{ext}} A^{\mu} .
$$

In (1), $A^{\mu}$ is the electromagnetic field and $m$ the mass of the Dirac field.

The equations of motion following from (1) are

$$
\begin{gathered}
\left(i \gamma^{\mu} \partial_{\mu}-e \gamma^{\mu} A_{\mu}-m\right) \psi=0, \\
\square A_{\mu}-\partial_{\mu} \partial^{\nu} A_{\nu}-e \bar{\psi} \gamma_{\mu} \psi=e j_{\mu}^{\text {ext }} .
\end{gathered}
$$

If we take a static, spherically symmetric source given by $[1,2]$

$$
j^{\mu}=\frac{Z \delta\left(r-r_{0}\right)}{r^{2}} \delta_{\mu 0}
$$

the vector potential $\boldsymbol{A}\left(A^{\mu}=\left(A_{0}, \boldsymbol{A}\right)\right)$ vanishes in the Coulomb gauge. We shall restrict ourselves to spherically symmetric solutions to eqs. (2), (3). This implies that we can only consider total spin $j$-values $j=1 / 2[3,4]$. Being interested in the lower-energy solution, we took $k \equiv \pm(j+1 / 2)=-1$. We then introduce

$$
\begin{aligned}
& \rho=m r, \\
& \Omega=\frac{\omega}{m},
\end{aligned}
$$




$$
\begin{gathered}
\psi=\exp [-i \Omega m t]\left(\begin{array}{c}
G(\rho) \mathscr{Y}_{j m}^{l}(\theta, \varphi) \\
i F(\rho) \sigma \cdot \widehat{r} \mathcal{Y}_{j m}^{l}(\theta, \varphi)
\end{array}\right), \\
f=\left(\frac{e A_{0}}{m}-\Omega\right) \rho
\end{gathered}
$$

$\mathscr{Y}_{j m}^{l}$ being the usual two-component spherical spinor. The ensuing equations of motion are then ( $\alpha$ is the fine structure constant)

$$
\begin{gathered}
F^{\prime}+\frac{2}{\rho} F-\left(1+\frac{f}{\rho}\right) G=0, \\
G^{\prime}-\left(1-\frac{f}{\rho}\right) F=0, \\
f^{\prime \prime}=-\alpha\left(F^{2}+G^{2}\right)+\frac{\alpha Z}{\rho_{0}} \delta\left(\rho-\rho_{0}\right) .
\end{gathered}
$$

Note that in the linear approximation (neglecting $F^{2}$ and $G^{2}$ ) eq. (11) yields

$$
f=-\alpha Z \theta\left(\rho-\rho_{0}\right)-\frac{\alpha Z}{\rho_{0}} \rho \theta\left(\rho_{0}-\rho\right),
$$

so that eqs. (9), (10) reduce to the usual Dirac equation for an electron in the attractive Coulomb field due to the charge density (4). From (9)-(11), one finds that the relevant boundary conditions at the origin are

$$
\begin{gathered}
G \underset{r \rightarrow 0}{\simeq} G_{0}, \\
F \underset{r \rightarrow 0}{\simeq} \frac{1+f_{0}}{3} G_{0} \rho, \\
f \underset{r \rightarrow 0}{\simeq} f_{0} \rho,
\end{gathered}
$$

while those at infinity are $\left(a=\sqrt{1-\Omega^{2}}\right)$

$$
\begin{gathered}
F \underset{r \rightarrow \infty}{\simeq} c \exp \left[-a_{p}\right], \\
G \underset{r \rightarrow \infty}{\simeq}-\sqrt{\frac{1-\Omega}{1+\Omega}} c \exp \left[-a_{p}\right], \\
f \underset{r \rightarrow \infty}{\simeq} \Omega_{p}+\alpha Q .
\end{gathered}
$$

The constants $G_{0}, f_{0}, c$ and $Q$ (the effective charge of the source) will be determined from the continuity of $G, F$ and $f$ at $\rho=\rho_{0}$, together with

$$
\left.f^{\prime}\right|_{\rho=\rho_{0}+\varepsilon}-\left.f^{\prime}\right|_{\rho=\rho_{0}-s}=\frac{\alpha Z}{\rho_{0}}
$$

which is a consequence of (12). 


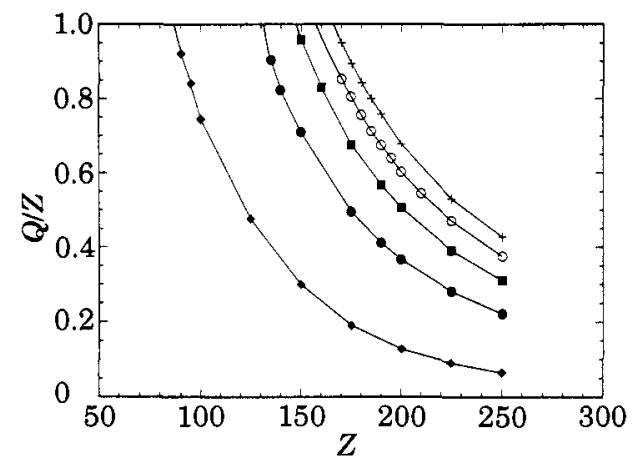

Fig. 1.

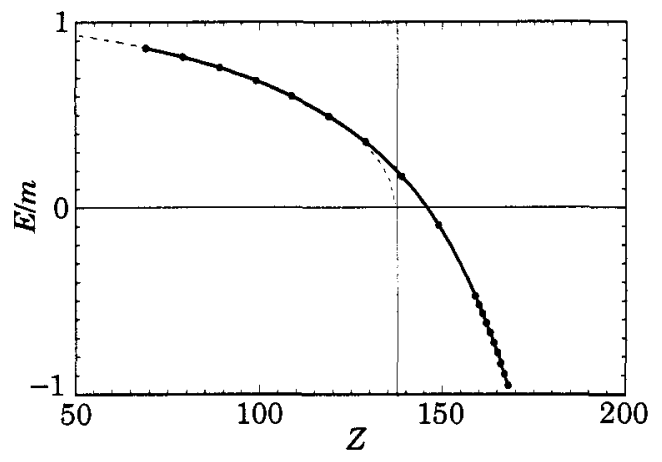

Fig. 2.

Fig. 1. - Ratio of the total charge $Q$ to the external charge $Z$ corresponding to partially screened solutions of eqs. (9)-(11), for various values of $\Omega:+\Omega=-0.783, \circ \Omega=-0.391, \square \Omega=0, \bullet \Omega=0.391$, $\Omega=0.783$. In this calculation, $m$ is equal to the electron mass and $r_{0}=7 \mathrm{fm}$.

Fig. 2. - Eigenvalues of the Dirac equation with Coulomb field corresponding to eq. (4), for $k=-1$ and $r_{0}=7 \mathrm{fm}$. The dashed curve corresponds to the point Coulomb problem $\left(r_{0}=0\right)$.

The energy $E$ associated with a given solution to the system (9)-(11) is [4]

$$
E=\int_{0}^{\infty}\left(\Omega m-e A_{0}\right)\left(F^{2}+G^{2}\right) \rho^{2} \mathrm{~d} \rho+\frac{1}{2 m \alpha} \int_{0}^{\infty}\left(e A_{0}^{\prime}\right)^{2} \rho^{2} \mathrm{~d} \rho .
$$

The value of the total charge $Q$ of the external source as a function of $Z$ is plotted in fig. 1 . One can see, comparing with fig. 2, that for any value of $\Omega, Q / Z$ reaches unity, when $Z$ is equal to the value at which the eigenvalue of the linear Dirac equation is equal to $\Omega$. Therefore, partial screening solutions exist for any value of $Z$. Moreover, fig. 3 shows that such solutions may have a lower energy than the pure Coulomb solution. We see that, when, for any value of $\Omega, Z$ reaches the value at which partial screening solution is possible, the energy $E$ corresponding to this solution first overcomes the Coulomb solution energy $E_{\mathrm{C}}$. Eventually, the energy becomes smaller than $E_{\mathrm{C}}$. Our results show that the value of $Z$ at
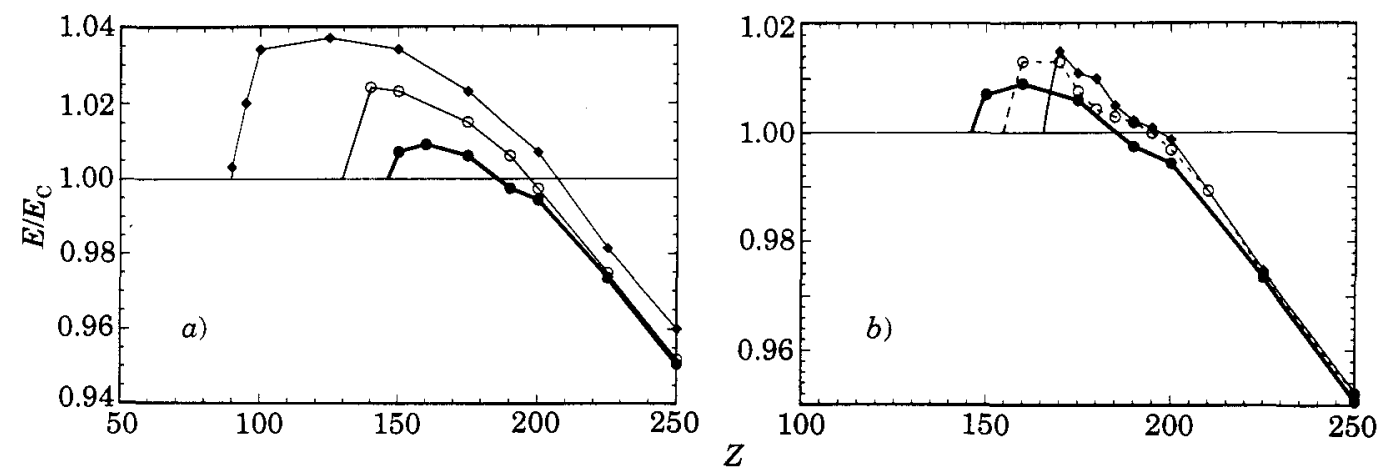

Fig. 3. - Ratio of the energy $E$ of the partial screened solution to that $E_{\mathrm{C}}$ of the pure Coulomb case, as a function of $Z$, for the same $\Omega$ values as in fig. 1. Part a) refers to $\Omega \geqslant 0: 0 \Omega 0,0 \Omega=0.391$, $\Omega=0.793$ and part $b$ ) to $\Omega \leqslant 0: \bullet \Omega=0, \circ \Omega=-0.391, \diamond \Omega=-0.783$. See text for detail. 


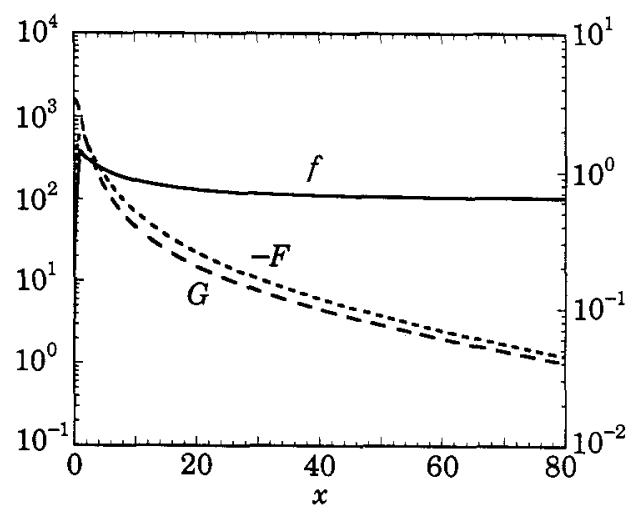

Fig. 4. - Fields $F$ and $G$ (scale on the left) and $f$ (scale on the right), as a function of $x=p_{p}$, for the nonlinear problem corresponding to eqs. (9)-(11), with $\Omega=0, Z=225$.

which a partially screened solution is energetically favoured occurs for $\Omega=0$, in similarity to the Klein-Gordon case. But, in contrast with the latter case, this does not happen at $Z_{0}$ (the value at which the eigenvalue of the linear problem vanishes). From our numerical computations, we can safely state that the value at which this occurs $(Z=180 \pm 5)$ is larger than $Z_{\mathrm{c}}$ (=168 in our numerical case). We therefore conclude that sources coupled with nonlinear classical Dirac fields are actually more "stable" than when coupled to scalar (Klein-Gordon) fields and that nonlinear effects of the kind studied here do not lower the value for the critical charge $Z_{\mathrm{c}}$ predicted by the Dirac equation.

We now wish to comment upon the accuracy of our numerical results. Solutions to eqs. (9)-(11) are constructed by minimizing the mismatch between the solutions obtained by integrating from $\rho=0$ to $\rho=\rho_{0}$ with conditions (12)-(14) and those obtained by integrating from $\rho=\infty$ (in practice, $\rho \approx 100 \rho_{0}$ ) down to $\rho=\rho_{0}$, in the space of the parameters $G_{0}, f_{0}, c$ and $Q$. It turns out that the minimum in the mismatch occurs in a region of the parameter space, where the topography of the relevant function is rather flat, whereas the energy depends rather sensitively upon the values of the parameters. The difficulty in the numerical integration is illustrated in fig. 4, which displays the fields $F, G$ and $f$. One can see that the asymptotic behaviour of $F$ and $G$ (eqs. (16), (17)) is barely achieved at eighty times the radius of the source and that the nonlinear coupling between $F, G$ and $f$ extends far outside the source. We estimate that the numerical error on $E / E_{\mathrm{C}}$ is of the order of one-fourth of a percent, which is largely sufficient to reveal the systematic behaviour of $E$ as a function of $Z$ and $\Omega$ and to assert our conclusions.

The work of MB was supported by the National Fund for Scientific Research (Belgium).

\section{REFERENCES}

[1] Bawin M. and Cugnon J., Phys. Rev. D, 28 (1983) 2091.

[2] Bawin M. and Cugnon J., Phys. Rev. D, 37 (1988) 2344.

[3] Ionescu D. C., ReinhardT J., Müller B., Greiner W. and Soff G., Phys. Rev. A, 38 (1988) 616.

[4] Ranada A. F. and Uson J. M., J. Math. Phys., 22 (1981) 2533.

[5] CALdi D. G., Comments Nucl. Part. Phys., 19 (1989) 137.

[6] Bawin M., Phys. Rev. D, 24 (1981) 3174. 